\title{
Andoyer construction for Hill and Delaunay variables
}

\author{
Jacques Laskar
}

Received: November 24, 2016/ Accepted: date

Abstract Andoyer variables are well known for the study of rotational dynamics. These variables were derived by Andoyer through a procedure that can be also used to obtain the Hill variables of the Kepler problem. Andoyer construction can also forecast the Delaunay variables which canonicity is then obtained without the use of a generating function.

Keywords Celestial mechanics · Delaunay variables $\cdot$ Hill variables · canonical transformations · Andoyer

\section{Introduction}

Buiding on the work of Binet (1841), Delaunay introduced the set of coordinates that he used for the elaboration of his Lunar theory (Delaunay, 1860). A few years later, in his thesis, Tisserand demonstrated how the canonicity of the Delaunay variables can be obtained through Hamilton-Jacobi theory (Tisserand, 1868). Tisserand's presentation is now ubiquitous in celestial mechanics textbooks. Here we present a derivation of the Delaunay variables that does not require HamiltonJacobi theory. We use the intermediate derivation of the Hill variables (Hill, 1913) following Andoyer (Andoyer, 1915, 1923). Transformations from Hill variables to Delaunay variables exist in the literature (Andoyer, 1913; Deprit, 1981, Floria, 1995 but they rely on a generating function which we will avoid here by using a direct computation based on the invariance of the canonical differential 2-form.

Henri Andoyer (1862-1929) is well-known for the derivation of the Andoyer variables that are very well adapted to rotational dynamics (Andoyer, 1915, 1923). In fact, the derivation of these variables is obtained through a very general procedure that can also be applied to the Keplerian two-body problem, and which then leads to the Hill variables (Andoyer, 1915, 1923).

Jacques Laskar

ASD, IMCCE-CNRS UMR8028, Observatoire de Paris, UPMC, 77 Av. Denfert-Rochereau, 75014-Paris, France E-mail: laskar@imcce.fr 


\section{Andoyer canonical criterion}

We recall here the criterion for canonicity of Andoyer (1923). Let us consider a $2 n$ dimensional phase with coordinates the canonical variables $\left(p_{j}, q_{j}\right)$, where $q_{j}$ are coordinate-type variables and $p_{j}$ the momenta. We then make the change of variables $\left(p_{j}, q_{j}\right) \longrightarrow\left(y_{k}, z_{k}\right)$ which we assume to be a good differentiable change of variables on the domain of interest, but without any assumption on its canonicity. We have

$$
\begin{aligned}
\sum_{j} p_{j} d q_{j} & =\sum_{j} p_{j}\left(\sum_{k} \frac{\partial q_{j}}{\partial y_{k}} d y_{k}\right)+\sum_{j} p_{j}\left(\sum_{l} \frac{\partial q_{j}}{\partial z_{l}} d z_{l}\right) \\
& =\sum_{k}\left(\sum_{j} p_{j} \frac{\partial q_{j}}{\partial y_{k}}\right) d y_{k}+\sum_{l}\left(\sum_{j} p_{j} \frac{\partial q_{j}}{\partial z_{l}}\right) d z_{l}
\end{aligned}
$$

A change of variable is canonical if it preserves the 2-form $\sigma_{2}=\sum_{j} d p_{j} \wedge d q_{j}$ (e.g. Arnol'd, 1989). This will be in particular the case if the change of variable preserves the 1 -form $\sigma_{1}=\sum_{j} p_{j} d q_{j}$, as $d\left(\sigma_{1}\right)=\sigma_{2}$. Thus, the transformations preserving the 1-form $\sum_{j} p_{j} d q_{j}$ form a subgroup of the canonical transformations. They are called Mathieu canonical transformations (Mathieu, 1874, Whittaker, 1904). For any variable $\alpha$, let us denote

$$
J_{\alpha}=\sum_{j} p_{j} \frac{\partial q_{j}}{\partial \alpha} .
$$

Equation (1) thus becomes

$$
\sum_{j} p_{j} d q_{j}=\sum_{k} J_{y_{k}} d y_{k}+\sum_{l} J_{z_{l}} d z_{l}
$$

Andoyer assumes that for all $k=1, \ldots, n, J_{y_{k}}=0$, and that for all $l=1, \ldots, n$, $J_{z_{l}}=u_{l}(y)$, where $\left(y_{k}\right)_{k=1, \ldots, n} \longrightarrow\left(u_{k}\right)_{k=1, \ldots, n}$ is a diffeomorphism. We have then

$$
\sum_{j} p_{j} d q_{j}=\sum_{j} u_{j} d z_{j}
$$

The change of variables $\left(p_{j}, q_{j}\right)_{j=1, \ldots, n} \longrightarrow\left(u_{j}, z_{j}\right)_{j=1, \ldots, n}$ preserves the 1 -form $\sum_{j} p_{j} d q_{j}$ and therefore preserves also the canonical 2 -form $\sum_{j} d p_{j} \wedge d q_{j}$. It is thus canonical. To search for such a canonical change of variables, one thus needs to compute $J_{\alpha}$ for any variable $\alpha$ in the set $\left\{y_{k}, z_{k}\right\}_{k=1, \ldots, n}$. Andoyer remarks then that $J_{\alpha}$ is the scalar product

$$
J_{\alpha}=\mathbf{p} \cdot \mathbf{V}_{\alpha}
$$

where $\mathbf{p}=\left(p_{j}\right)$ is the momentum vector, and the virtual velocity $\mathbf{V}_{\alpha}=\left(\frac{\partial q_{j}}{\partial \alpha}\right)$ is obtained when only varying the variable $\alpha$ in the position vector $\left(q_{j}\right)$. When the Andoyer criterion is verified, the quantities $J_{\alpha}$ will be the new conjugate momentum associated to the coordinate-type variables $\alpha$. 


\section{Hill variables}

For a celestial body $P$, we consider the Kepler problem in a fixed reference frame $(\mathbf{i}, \mathbf{j}, \mathbf{k})$, whose origine $O$ coincides with the attractive center, with radius vector $\mathbf{r}=r \mathbf{u}$, velocity $\dot{\mathbf{r}}$, gravitational coupling parameter $\mu$, and Hamiltonian per unit of mass

$$
\mathcal{H}=\frac{1}{2} \dot{\mathbf{r}}^{2}-\frac{\mu}{r} .
$$

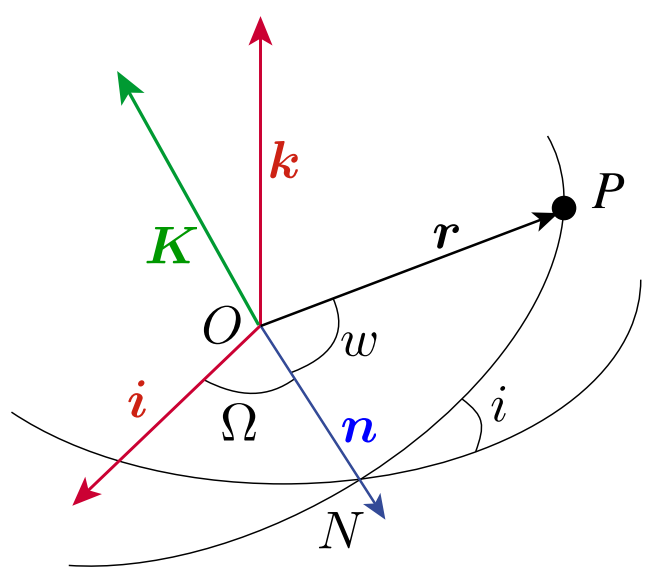

Fig. 1 Reference frame and notations. The vectors $\mathbf{i}, \mathbf{k}, \mathbf{K}, \mathbf{n}$ are unit vectors. $N$ is the ascending node.

The orbital plane $(\mathbf{r}, \dot{\mathbf{r}})$ is orthogonal to the angular momentum per unit mass $\mathbf{G}=G \mathbf{K}(G=\|\mathbf{G}\|)$, and is defined by the longitude of the ascending node $\Omega$ and inclination $i$ (Fig.1). The position of the celestial body $P$ is defined when $r$ and the argument of latitude $w=\omega+v$ ( $\omega$ is the argument of perihelion, and $v$ the true anomaly) are given. We have thus characterized the position of $P$ with the four variables $(r, \Omega, w, i)$. With Hamiltonian (2), the momentum vector is simply the velocity $\dot{\mathbf{r}}$. Following Andoyer, we extend the transformation $(\dot{\mathbf{r}}, \mathbf{r}) \longrightarrow(r, \Omega, w, i)$ into a genuine canonical change of variables. For this, we evaluate all $\mathbf{V}_{\alpha}$ and $J_{\alpha}=\dot{\mathbf{r}} \cdot \mathbf{V}_{\alpha}$ quantities (5). We remind that when rotating a vector $\mathbf{A}$ around a fixed unit vector $\mathbf{b}$ by an angle $\theta$, we have

$$
\frac{d \mathbf{A}}{d \theta}=\mathbf{b} \wedge \mathbf{A}
$$

The virtual velocity $\mathbf{V}_{i}$ is obtained from a rotation of angle $i$ around $\mathbf{n}$, the unit vector in the direction of $\mathbf{O N}$ (Fig.1). We have thus

$$
\mathbf{V}_{i}=\mathbf{n} \wedge \mathbf{r}, \quad J_{i}=\dot{\mathbf{r}} \cdot \mathbf{V}_{i}=0 .
$$

In a similar way, we have

$$
\begin{array}{ll}
\mathbf{V}_{w}=\mathbf{K} \wedge \mathbf{r}, & J_{w}=\dot{\mathbf{r}} \cdot(\mathbf{K} \wedge \mathbf{r})=\mathbf{K} \cdot(\mathbf{r} \wedge \dot{\mathbf{r}})=G \\
\mathbf{V}_{\Omega}=\mathbf{k} \wedge \mathbf{r}, & J_{\Omega}=\dot{\mathbf{r}} \cdot(\mathbf{k} \wedge \mathbf{r})=\mathbf{k} \cdot(\mathbf{r} \wedge \dot{\mathbf{r}})=G \cos i
\end{array}
$$


and as $\mathbf{r}=r \mathbf{u}$

$$
\mathbf{V}_{r}=\mathbf{u}, \quad J_{r}=\dot{\mathbf{r}} \cdot \mathbf{u}=(\dot{r} \mathbf{u}+r \dot{\mathbf{u}}) \cdot \mathbf{u}=\dot{r} .
$$

Moreover, as $\mathbf{r}$ depends only on $r, w, \Omega, i$ and not on $\dot{r}$ or $G$, we have $\mathbf{V}_{\dot{r}}=\mathbf{V}_{G}=0$, and then $J_{\dot{r}}=J_{G}=0$. We are thus in the framework of the application of Andoyer criterion and we can conclude that the change of variables

$$
(\dot{\mathbf{r}} ; \mathbf{r}) \longrightarrow(\dot{r}, G, G \cos i ; r, w, \Omega)
$$

is canonica ${ }^{1}$ In these new variables, known as the Hill variables (Hill, 1913), the Hamiltonian becomes 2

$$
\mathcal{H}=\frac{1}{2}\left(\dot{r}^{2}+\frac{G^{2}}{r^{2}}\right)-\frac{\mu}{r} .
$$

The Hamiltonian 12 has only one degree of freedom $(\dot{r}, r)$ (and one parameter $G)$. It is thus obviously integrable but not in a simple way. To fully achieve the reduction of the problem to a trivially integrable Hamiltonian, we will use the Delaunay variables.

\section{Delaunay variables}

We assume that we have already obtained the classical orbital elements $(a, e, i, M, \omega, \Omega)$ for an elliptical orbit, with the above definitions for $i, \omega, \Omega$ (Fig.1). The derivation of $(a, e, M)$ is given in the Appendix. The mean anomaly $M$ is the angle that is proportional to the area swept by $\mathbf{r}$ from perihelion. In the two-body problem, we will thus have $d M / d t=n$, where $n=2 \pi / T$ is the mean motion (see the Appendix) with $n^{2} a^{3}=\mu$. The variable $L$ was introduced by Delaunay (1860). For an elliptical orbit, we have $\mathcal{H}=-\mu / 2 a . L$ is therefore defined as the only ${ }^{3}$ function $L(a)$ such that

$$
\frac{d M}{d t}=n=\frac{d \mathcal{H}}{d L}=\mu^{1 / 2} a^{-3 / 2}
$$

which implies

$$
\frac{d L}{d a}=\frac{\sqrt{\mu}}{2 \sqrt{a}}
$$

and thus $L=\sqrt{\mu a}$, up to an additive constant. The mean anomaly $M$ defines the body position from perihelion. We will add $\omega$ and $\Omega$ as angular variables to define the position of the perihelion. Following the previous section, we can use Andoyer's ideas to complete the change of variables into a canonical change of

1 An alternate approach following S. Breiter (2016, pers. comm.) and inspired by (Deprit and Elipe 1993) could be purely analytical, with a direct computation of the canonical 1-form, gathering the derivations of equations 89 ). Indeed, we have (Fig.1)

$$
d \mathbf{r}=\mathbf{u} d r+(\mathbf{k} \wedge \mathbf{r}) d \Omega+(\mathbf{n} \wedge \mathbf{r}) d i+(\mathbf{K} \wedge \mathbf{r}) d w
$$

thus

$$
\dot{\mathbf{r}} d \mathbf{r}=\dot{r} d r+G d w+G \cos i d \Omega .
$$

2 As $\mathbf{u}$ is a unit vector, $\mathbf{u} \perp \dot{\mathbf{u}}$, and thus $\dot{\mathbf{r}}^{2}=\dot{r}^{2}+r^{2}\|\dot{\mathbf{u}}\|^{2}$, and $G=r^{2}\|\dot{\mathbf{u}}\|$.

3 Up to an additive constant. 
variables. For $M$ fixed, varying $\omega$ is equivalent of varying $w$, and thus, as in section 3 , we have $J_{\omega}=G$. Therefore, $G$ is naturally conjugate to $\omega$, and as previously for Hill variables, $J_{\Omega}=H$. We have thus obtained the Delaunay variables in a natural way, following Andoyer ideas. We have

$$
\begin{aligned}
L & =\sqrt{\mu a}, & & M, \\
G & =L \sqrt{1-e^{2}}, & & \omega, \\
H & =G \cos i, & & \Omega .
\end{aligned}
$$

To prove that the change of variable from Hill variables to Delaunay variables is symplectic, we consider the 1 -form

$$
\sigma=\dot{r} d r+G d w+H d \Omega-(L d M+G d \omega+H d \Omega),
$$

which reduces to (with $w=v+\omega)$

$$
\sigma=\dot{r} d r+L \sqrt{1-e^{2}} d v-L d M .
$$

In order to evaluate this expression, we use the differential relations of the Appendix (Eq 32 which allow to express $\sigma$ in term of $E, e, a, d E, d e, d a$ as

$$
\sigma=2 e \sqrt{\mu a} \cos E d E+2 \sqrt{\mu a} \sin E d e+\sqrt{\frac{\mu}{a}} e \sin E d a .
$$

The 1-form $\sigma$ is not null. So the change of variable from Hill variables (and thus as well from cartesian coordinates) to Delaunay variables is not a Mathieu transformation. Nevertheless, a simple derivate of $(\mathrm{Eq} 18)$ gives

$$
d \sigma=0
$$

Thus

$$
d \dot{r} \wedge d r+d G \wedge d w+d H \wedge d \Omega=d L \wedge d M+d G \wedge d \omega+d H \wedge d \Omega,
$$

and the change of variable to Delaunay variables is canonical. In these variables $(L, G, H, M, \omega, \Omega)$, the Hamiltonian 2,12 becomes trivially integrable as

$$
\mathcal{H}=-\frac{\mu^{2}}{2 L^{2}} \text {. }
$$

\section{Discussion}

The Andoyer construction exposed here allowed to introduce in a natural way the Delaunay variables. The variables $G$ and $H$ appear as the conjugate variables to $\omega$ and $\Omega$ through Andoyer derivation. This approach can be compared to the derivation of (Bilimovitch, 1943 Broucke, 1978) which share features with Andoyer's approach. The main difference, apart from using the Hill variables as an intermediary step here, is the absence of the need for generating function by relying on the conservation of the canonical 2-form. Finally, it should be said that

4 Broucke $\sqrt{1978)}$ shows that $\sigma=2 d(r \dot{r})$ in 18 , and thus $2 r \dot{r}$ can be considered as a generating function for the transformation to Delaunay variables. This adds some information, but it is not clear how this generating function can be obtained in a natural way from 18 . 
other authors have searched for derivations of the Delaunay variables that avoid Hamilton-Jacobi theory or generation functions. Brouwer and Clemence (1961) compute directly all the Lagrange brackets of the Delaunay variables while more geometrical approaches are provided by (Chang and Marsden, 2003; Féjoz, 2013).

Acknowledgements The author thanks A. Albouy and L. Floría for historical insights, and S. Breiter for pointing out some inconsistency in the first version of this paper.

\section{Appendix : Differential relations in elliptical elements}

In order to be self-consistent, here are briefly reminded some derivation of the classical elliptical elements that are used in the present work. The Hamiltonian of the two-body problem is 2 ,

$$
\mathcal{H}=\frac{1}{2} \dot{\mathbf{r}}^{2}-\frac{\mu}{r}
$$

with the associated Newton equation

$$
\ddot{\mathbf{r}}=-\nabla_{\mathbf{r}} \mathcal{H}=-\mu \frac{\mathbf{r}}{r^{3}} .
$$

By direct computation, we have $d \mathcal{H} / d t=0, d \mathbf{G} / d t=0$ where $\mathbf{G}=\mathbf{r} \wedge \dot{\mathbf{r}}$ is the angular momentum, ans also $d \mathbf{P} / d t=0$, where $\mathbf{P}$ is the Laplace-Runge vector

$$
\mathbf{P}=\frac{\dot{\mathbf{r}} \wedge \mathbf{G}}{\mu}-\mathbf{u}
$$

where $\mathbf{u}$ is the unit vector $\mathbf{r} / r$ of angle $v$ with $\mathbf{P}$. We have thus

$$
\mathbf{P} \cdot \mathbf{u}=e \cos v=\frac{\mathbf{r} \cdot(\dot{\mathbf{r}} \wedge \mathbf{G})}{r \mu}-1=\frac{G^{2}}{\mu r}-1,
$$

where $e$ (the eccentricity) is the norm of $\mathbf{P}$. With $e<1$, we thus obtain that the orbit is an ellipse of semi-major axis $a$, and eccentricity $e$, with $G=\sqrt{\mu a\left(1-e^{2}\right)}$ and polar equation

$$
r=\frac{a^{2}\left(1-e^{2}\right)}{1+e \cos v}
$$

As $\mathcal{H}$ is constant, we can compute its value for $v=0$. We have then $\dot{r}=0, r=a(1-e)$, and thus $\mathcal{H}=-\mu / 2 a$. The area $\mathcal{A}$ of the ellipse is

$$
\mathcal{A}=\pi a^{2} \sqrt{1-e^{2}}=\int_{0}^{2 \pi} \frac{1}{2} r^{2} d v=\int_{0}^{T} \frac{1}{2} G d t=\frac{G T}{2},
$$

where $T$ is the period of the motion. The mean motion $n=2 \pi / T$ then verifies the third Kepler's law $n^{2} a^{3}=\mu$. We need also to introduce the eccentric anomaly $E$ defined as the angle $F O P^{\prime}$ where $P^{\prime}$ is the point on the circle of radius $a$ obtained by affininity of ration $1 / \sqrt{1-e^{2}}$ from the ellipse (Fig 2).

If we denote $M$ (the mean anomaly) the angle proportional to the area $\mathcal{A}(A F P)$, we have $\mathcal{A}\left(A F P^{\prime}\right)=\mathcal{A}(A F P) / \sqrt{1-e^{2}}=a^{2} M / 2$. the relation among areas $\mathcal{A}\left(A O P^{\prime}\right)=\mathcal{A}\left(A F P^{\prime}\right)+$ $\mathcal{A}\left(F O P^{\prime}\right)$ then gives the Kepler equation

$$
M=E-e \sin E .
$$




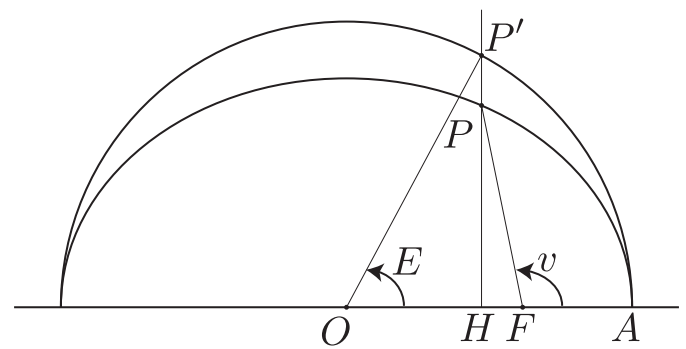

Fig. 2 The circle is obtained from the ellipse by affinity of ration $1 / \sqrt{1-e^{2}}$ from the ellipse. $v$ is the true anomaly, and $E$ the eccentric anomaly.

In the reference frame $F, \mathbf{I}, \mathbf{J}$, with $\mathbf{I}$ the unit vector along $\mathbf{F A}$, the position and velocity $(\mathbf{r}, \dot{\mathbf{r}})$ have coordinates $(X, Y),(\dot{X}, \dot{Y})$ with

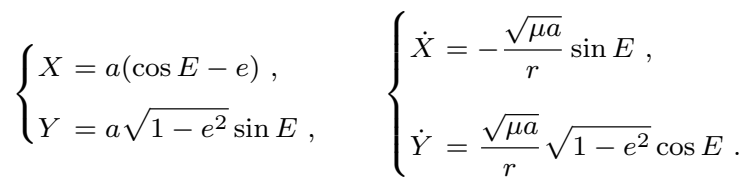

with

As $\mathbf{r} \cdot \dot{\mathbf{r}}=r \dot{r}$, we have also

$$
r=a(1-e \cos E) .
$$

$$
\dot{r}=\frac{\sqrt{\mu a}}{r} e \sin E .
$$

All relations 26, 28, 30, 31) are geometrical relations in the phase space of $(\mathbf{r}, \dot{\mathbf{r}})$. All quantities $v, M, r, \dot{r}$ can be expressed in term of $E, e, a$. By simple differentiation, we obtain the differential relations

$$
\begin{aligned}
& d M=\frac{r}{a} d E-\sin E d e, \\
& d v=\frac{a}{r} \sqrt{1-e^{2}} d E+\frac{a}{r \sqrt{1-e^{2}}} \sin E d e, \\
& d r=a e \sin E d E-a \cos E d e+\frac{r}{a} d a,
\end{aligned}
$$

with $r / a=1-e \cos E$.

\section{References}

Andoyer H (1913) Sur l'anomalie excentrique et l'anomalie vraie comme éléments canoniques du mouvement eliptique, d'après MM. T. Levi-Civita et G.-W. Hill. Bulletin Astronomique, Série I 30:425-429

Andoyer H (1915) Sur les problèmes fondamenteaux de la mécanique céleste. Bulletin Astronomique, Serie I 32:5-18

Andoyer H (1923) Cours de mécanique céleste, Vol. I. Gauthier-Villars, Paris

Arnol'd VI (1989) Mathematical Methods of Classical Mechanics. Springer

Bilimovitch A (1943) Über die Anwendungen der Pfaffschen Methode in der Störungsthorie. Astronomische Nachrichten 273:161 
Binet M (1841) Mémoire sur la variation des constantes arbitraires dans les formules générales de la dynamique, et dans un système d'équations analogues plus étendues. Journal de l'Ecole Polytechnique 28, T.XVII:1-94

Broucke R (1978) On Pfaff's equations of motion in dynamics - Applications to satellite theory. Celestial Mechanics 18:207-222

Brouwer D, Clemence G (1961) Methods of Celestial Mechanics. Academic Press, New York

Chang D, Marsden J (2003) Geometric derivation of the delaunay variables and geometric phases. Celestial Mechanics and Dynamical Astronomy 86:185-208

Delaunay C (1860) Théorie du mouvement de la Lune, Vol. I. Mémoires de l'Académie des Sciences de l'Institut Impérial de France XXVIII:1-883

Deprit A (1981) The elimination of the parallax in satellite theory. Celestial Mechanics 24:111153

Deprit A, Elipe A (1993) Complete reduction of the Euler-Poinsot problem. Journal of the Astronautical Sciences 41:603-628

Féjoz J (2013) On action-angle coordinates and the Poincaré coordinates. Regular \& Chaotic Dynamics 18(6):703-718

Floria L (1995) A simple derivation of the hyperbolic Delaunay variables. The Astronomical Journal 110:940

Hill GW (1913) Motion of a system of material points under the action of gravitation. The Astronomical Journal 27:171-182

Mathieu E (1874) Mémoire sur les équations différentielles canoniques de la mécanique. Journal de Mathématiques Pures et Appliquées (Journal de Liouville) XIX:265-306

Tisserand F (1868) Exposition, d'après les principes de Jacobi, de la méthode suivie par M. Delaunay dans sa théorie du Mouvement de translation de la Lune, Thèse de doctorat. Gauthier-Villars (Paris)

Whittaker E (1904) A treatise on the analytical dynamics of particles and rigid bodies. Cambridge Univ. Press, London 